\title{
Bioinformatic analysis of microRNA and mRNA Regulation in peripheral blood mononuclear cells of patients with chronic obstructive pulmonary disease
}

Xiaomin Dang ${ }^{2 \dagger}$, Xiaoyan $\mathrm{Qu}^{1+}$, Weijia Wang ${ }^{1}$, Chongbing Liao ${ }^{1}$, Ying $\mathrm{Li}^{1}$, Xiaojin Zhang ${ }^{1}$, Dan $\mathrm{Xu}^{1}$, Carolyn J. Baglole ${ }^{3}$, Dong Shang ${ }^{2^{*}}$ and Ying Chang ${ }^{1 *}$

\begin{abstract}
Background: Chronic obstructive pulmonary disease (COPD) is a progressive, irreversible chronic inflammatory disorder typified by increased recruitment of monocytes, lymphocytes and neutrophils. Because of this, as well as the convenience of peripheral blood nuclear cells (PBMCs) assessments, miRNA profiling of PBMCs has drawn increasing attention in recent years for various disease. Therefore, we analyzed miRNA and mRNA profiles to understand their regulatory network between COPD subjects versus smokers without airflow limitation.

Methods: miRNA and mRNA profiling of PBMCs from pooled 17 smokers and 14 COPD subjects was detected by high-throughput microarray. The expression of dysregulated miRNAs were validated by q-PCR. The miRNA targets in dysregulated mRNAs were predicted and the pathway enrichment was analyzed.

Results: miRNA microarray showed that 8 miRNAs were up-regulated and 3 miRNAs were down-regulated in COPD subjects compared with smokers; the upregulation of miR-24-3p, miR-93-5p, miR-320a and miR-320b and the downregulation of miR-1273 g-3p were then validated. Bioinformatic analysis of regulatory network between miRNA and mRNA showed that NOD and TLR were the most enriched pathways. miR-24-3p was predicted to regulate IL-18, IL-1ß, TNF, CCL3 and CCL4 and miR-93-5p to regulate IkBa.

Conclusions: The expression of miRNA and mRNA were dysregulated in PBMCs of COPD patients compared with smokers without airflow limitation. The regulation network between the dysregulated miRNA and mRNA may provide potential therapeutic targets for COPD.
\end{abstract}

Keywords: miRNA, COPD, PBMC, Microarray, MicroRNA

\section{Background}

Chronic obstructive pulmonary disease (COPD) is a progressive, irreversible chronic inflammatory disorder. Caused predominantly by cigarette smoking, COPD is one of the leading causes of mortality globally [1].

\footnotetext{
* Correspondence: sd74@vip.sina.com; changyingcn@hotmail.com ${ }^{\dagger}$ Equal contributors

2Department of Respiration, The First Affiliated Hospital, Xi'an Jiaotong University, Xi'an 710061, Shaanxi province, China

${ }^{1}$ Center for Translational Medicine, The Key Laboratory of Biomedical Information Engineering of Ministry of Education, School of Life Science and Technology and Frontier Institute of Science and Technology, Xi'an Jiaotong University, Xi'an 710049, Shaanxi province, China

Full list of author information is available at the end of the article
}

Although cigarette smoking is the major cause of COPD, there is currently no satisfactory therapy to treat individuals once the disease is established. Inflammation plays a pivotal role in the disease process, with $\mathrm{CD}^{+} \mathrm{T}$ lymphocytes, neutrophils and macrophages being the main type of immune cells of local inflammatory milieu of COPD [2]. Different immunoregulatory properties of $\mathrm{T}$ cells and monocytes have been demonstrated in COPD patients [3], and the inflammatory response in the lungs of COPD patients is strongly linked to tissue destruction and alveolar airspace enlargement [4], in part due to the loss of lung structural cells due to heightened apoptosis. 
microRNAs (miRNAs) are a growing family of small non-coding RNAs (approximately 19 to 25 nucleotides long) with a regulatory function on gene expression [5]. Through binding to the $3^{\prime}$ untranslated region (3' UTR) of target messenger RNAs, miRNAs can lead to direct inhibition of protein translation or degradation of messenger RNA [5]. In addition, miRNAs can alter gene expression by targeting transcription factors and DNA methyltransferases. In this way, miRNAs work as posttranscriptional regulators of gene expression and control various cellular processes such as differentiation, proliferation, and cell-cell interaction. The dysregulation of miRNAs is linked to a wide spectrum of diseases, including proliferative vascular disease, cardiac disorders, lung diseases, kidney diseases, diabetes mellitus, fibrosis and cancer [6-8].

A few studies have been conducted to reveal the dysregulation and role of miRNAs in COPD. Ezzie et al. [9] compared the miRNA expression in lung tissue derived from smokers with and without COPD and identified 70 differentially expressed miRNAs. A report by Pottelberge et al. [10] demonstrated that 34 miRNAs were differentially expressed between never-smokers and current smokers without airflow limitation, and 8 miRNAs were expressed at a significantly lower level in current-smoking patients with COPD compared with never-smokers without airflow limitation. Another study showed that microRNA-34c is associated with emphysema severity in COPD [11]. Sato et al. [12] observed reduced miR-146a expression in lung fibroblasts of patients with COPD and showed that miR-146a deficiency increased the expression of $\mathrm{PGE}_{2}$ through depression of the miR-146a target COX-2. Finally, Lewis et al. [13] found downregulated miR-1 expression in quadriceps muscles and speculated that this is linked to the muscle weakness observed in COPD.

On account of the convenience of peripheral blood nuclear cells (PBMC) assessments, the miRNA profiling of $\mathrm{PBMC}$ has drawn increasing attention in recent years in various diseases such as cancer [14, 15], Alzheimer's Disease [16], diabetes [17] and autoimmune disease [18]. However, there is no report regarding miRNA expression profiles of PBMC in COPD patients. Therefore, in this study, we sought to determine if miRNAs were differentially expressed in PBMCs of COPD patients and if miRNA expression may be linked to dysregulated mRNA expression relevant to the pathogenesis of COPD. We analyzed miRNA and mRNA expression profiles in PBMCs from COPD patients versus smokers without airflow limitation. The mRNA targets of the dysregulated miRNA were predicted and pathway enrichment was analyzed. We identified a signature of COPD-associated miRNA, such that miR-24-3p, miR93-5p, miR-320a, miR-320b and miR-1273g-3p were significantly dysregulated in COPD patients. Bioinformatic analysis between miRNA and predicted mRNA showed that NOD and TLR were the most enriched pathways. In NOD pathway, miR-24-3p was predicted to regulate IL18/IL1B/TNF, and miR-93-5p to regulate NLRP3/IL6/NFKBIA. In TLR pathway, miR-24-3p was predicted to regulate CCL3/CCL4/IL1B/TNF, and miR93-5p to regulate IL6/CXCL10/NFKBIA.

\section{Methods \\ Subjects}

Peripheral venous blood was taken in heparin-coated tubes from 17 smokers without airflow limitation and 14 COPD subjects at Department of Respiration, The First Affiliated Hospital, Xi'an Jiaotong University, Xi'an, China. COPD subjects were eligible for this study if they met the following criteria: age $\geq 50$ and $\leq 76$ years; smoking history ( $\geq 20$ pack-years); post-bronchodilator $\mathrm{FEV}_{1} \geq 25 \%$ of predicted value and post-bronchodilator $\mathrm{FEV}_{1} /$ forced vital capacity $(\mathrm{FVC}) \leq 0.70$; no history of asthma, atopy (as assessed by an allergy skin prick test during screening) or any other active lung disease. Patients on home oxygen or with raised carbon dioxide tension $(>44 \mathrm{mmHg}), \alpha_{1}$-antitrypsin deficiency, recent exacerbation (in the last 4 weeks), an uncontrolled medical condition or hypersensitivity to inhaled corticosteroids and bronchodilators were not eligible for the study. All smokers without airflow limitation met the following criteria: age $\geq 42$ and $\leq 75$ years, Post-BD FEV1\% predicted $>80$, no diagnostic cancer, diabetes, cardiovascular disease and hypertension, no use of inhaled or oral corticosteroids in the previous 6 months, no atopy, and no respiratory tract infection 1 month prior to the study. Patient characteristics are in Table 1 . The experimental procedures were performed with ethical approval from the Research Ethics Boards of The First Affiliated Hospital, Xi'an Jiaotong University (2015-015).

Table 1 Clinical characteristics of smokers without airflow limitation and COPD patients

\begin{tabular}{lll}
\hline & Smokers & COPD \\
\hline Number & 17 & 14 \\
Age & $56 \pm 17$ & $69 \pm 8$ \\
Male/Female & $17 / 0$ & $13 / 1$ \\
Current/ex-smokers & $14 / 3$ & $8 / 6$ \\
Pack-years & $23 \pm 3$ & $27 \pm 6$ \\
Post-BD FEV1\% predicted & $92.9 \pm 9.0$ & $29.0 \pm 10.6$ \\
GOLD Stage & & \\
\multicolumn{1}{|l|}{ II } & - & 0 \\
III-IV & - & 2 \\
\hline
\end{tabular}

Data are presented as Means \pm SD. BD bronchodilator, FEV1 forced expiratory volume in $1 \mathrm{~s}$ 


\section{PBMC isolation and RNA extraction}

PBMCs were isolated from venous blood by density gradient centrifugation over Ficoll-Paque PLUS reagent (GE Healthcare, Uppsala, Sweden) and suspended in QIAzol Lysis Reagent (Qiagen, Dusseldorf, Germany). Total RNA was extracted using miRNeasy Mini Kit (Qiagen) according to the manufacturer's procedure. RNA integrity was determined by formaldehyde denaturing gel electrophoresis.

\section{miRNA and gene expression microarray}

Equal amount of RNA sample from each smokers $(N=17)$ and COPD patients $(N=14)$ was pooled respectively for miRNA profiling assay using Affymetrix GeneChip miRNA Array v.4.0 (Affymetrix, Santa Clara, CA, USA) by Capitalbiotech company, Beijing, China. Briefly, after labeled with Biotin, the total RNA was subsequently hybridized overnight. The GeneChip miRNA 4.0 arrays, containing 30,424 total mature miRNA probe sets including 2,588 mature human miRNAs and miRNAs of 202 other organisms, were washed and stained using the Affymetrix GeneChip Hybridization Wash and Stain Kit and were then scanned with the Affymetrix GeneChip Scanner 3000 (Affymetrix, Santa Clara, CA, USA).

\section{Messenger RNA microarray}

Samples were prepared for mRNA microarray analysis using Agilent Human Gene Expression Microarray V4.0 (Santa Clara, California, USA). Hybridized slides were then washed and scanned with Agilent Microarray Scanner System (G2565CA).

\section{Data analysis}

The miRNA and mRNA array data were analyzed for data summarization, normalization and quality control using GeneSpring V11.5 software (Agilent). To select differentially expressed genes, we used threshold values of $>2$ fold change. The data were Log2 transformed and median centred by genes using the Adjust Data function of CLUSTER 3.0 software. Further analysis was performed by hierarchical clustering with average linkages. Finally, we performed tree visualization using Java Treeview (Stanford University School of Medicine, Stanford, CA, USA).

\section{Quantitative reverse transcription PCR validation}

Independent assays were performed using quantitative reverse transcription PCR (qRT-PCR) on all patient samples for individual miRNA (miR-24-3p, miR-93-5p, miR-320a, miR-320b, miR-191-5p, let-7b-5p, miR-3423p, miR-92a-3p, miR-3613-3p, miR-1273 g-3p and miR4668-5p) (Qiagen) and predicted target genes (IL18, IL1B, TNF, CCL3, CCL4, NLRP3, IL6, NFKBIA and
CXCL10) (Bio-rad, Foster city, USA). In addition, the expression of miR-24-3p, miR-93-5p, miR-320a, miR$320 \mathrm{~b}$ and miR-1273 g-3p was detected on the isolated different cell types including $\mathrm{CD} 4^{+} \mathrm{T}$ cells, $\mathrm{CD} 8^{+} \mathrm{T}$ cells, $\mathrm{CD} 20^{+} \mathrm{B}$ cells and $\mathrm{CD} 14^{+}$monocytes from PBMCs in some COPD patients by positive selection (Anti-PE MicroBeads UltraPure, Miltenyi Biotec, Teterow, Germany). Data were presented relative to U6 for miRNA and $\beta$-actin for target genes based on calculations of $2^{(-\sigma \sigma C t)}$. The primer sequences for target genes were listed in Table 2. Statistical significance was defined as $p<0.05$ as measured by the $t$ test using GraphPad Prism 5 software (GraphPad, San Diego, CA, USA).

\section{Target prediction and network analysis}

The target genes for miRNAs were predicted using miRanda, MirTarget2, PicTar, PITA and TargetScan. The regulation network diagram between miRNAs and mRNAs was generated using Cytoscape. Based on the data of mRNA array, the predicted target genes that negatively regulated by the validated dysregulated miRNAs were selected for the further pathway enrichment analysis. The DAVID [19] online analysis tool was used and the significant enrichment threshold was $P$ value of Modified Fisher exact less than 0.05 and enriched gene count more than 2 .

Table 2 The sequence of primers for real-time PCR

\begin{tabular}{|c|c|c|}
\hline Gene & Sequence $\left(5^{\prime}-3^{\prime}\right)$ & Direction \\
\hline \multirow[t]{2}{*}{ IL18 } & GACCAAGGAAATCGGCCTCTA & Forward \\
\hline & AGTTACAGCCATACCTCTAGGC & Reverse \\
\hline \multirow[t]{2}{*}{ IL1B } & CTCTGGGATTCTCTTCAGCCA & Forward \\
\hline & AATAAGCCATCATTTCACTGGCG & Reverse \\
\hline \multirow[t]{2}{*}{ TNF } & GCTGCACTTTGGAGTGATCG & Forward \\
\hline & GGCCAGAGGGCTGATTAGAG & Reverse \\
\hline \multirow[t]{2}{*}{ CCL3 } & GCTCTCTGCAACCAGTTCTCT & Forward \\
\hline & GGCTGCTCGTCTCAAAGTAGT & Reverse \\
\hline \multirow[t]{2}{*}{ CCL4 } & CTCCCAGCCAGCTGTGGTATTC & Forward \\
\hline & CCAGGATTCACTGGGATCAGC & Reverse \\
\hline \multirow[t]{2}{*}{ NLRP3 } & ATGAGAGTGTTGTGTGAAACGC & Forward \\
\hline & GAGATGTCGAAGCAGCACTCA & Reverse \\
\hline \multirow[t]{2}{*}{ IL6 } & CAGACAGCCACTCACCTCTTC & Forward \\
\hline & CAGGTTGTITTCTGCCAGTGC & Reverse \\
\hline \multirow[t]{2}{*}{ NFKBIA } & GTACGAGCAGATGGTCAAGGA & Forward \\
\hline & GGTCAGTGCCTITTCTTCATGG & Reverse \\
\hline \multirow[t]{2}{*}{ CXCL10 } & GCCATTCTGATTTGCTGCCTT & Forward \\
\hline & ACTAATGCTGATGCAGGTACAG & Reverse \\
\hline \multirow[t]{2}{*}{$\beta$-actin } & TACCTCATGAAGATCCTCACC & Forward \\
\hline & TTTCGTGGATGCCACAGGAC & Reverse \\
\hline
\end{tabular}




\section{Results}

MicroRNAs are dysregulated in PBMCs of COPD patients We investigated the miRNA profiling in pooled PBMCs from 17 smokers without airflow limitation and 14 COPD patients (Fig. 1). Compared with smokers, there were 103 up-regulated and 34 down-regulated miRNAs in COPD patients. We selected the dysregulated miRNAs with differences in the fluorescence intensity higher than 1000 between the smokers and COPD patients. As shown in Table 3, there were a total of 8 up-regulated and 3 down-regulated miRNA; these were selected for the further validation and analysis.

\section{Validation of dysregulated miRNAs in PBMCs of COPD patients}

Among the selected 11 dysregulated miRNAs in COPD patients versus smokers, we validated the expression of 5 miRNAs (miR-24-3p, miR-93-5p, miR-320a, miR-320b and miR-1273 g-3p) in smokers and COPD patients by qRT-PCR, as shown in Fig. 2a. MiR-1273 g-3p was the most highly expressed miRNA in PBMCs of smokers, while miR-320a and miR-320b had the higher relative abundance in PBMCs of COPD patients (Fig. 2b). The correlation analysis between miRNA expression and FEV1\% predicted showed that significant negative relevance appeared in the expression of miR-24-3p, miR$320 \mathrm{a}$ and miR-320b and positive relevance appeared in miR-1273 g-3p (Fig. 2c). However, there is no difference
Table 3 Selected dysregulated miRNAs in COPD patients compared with smokers without COPD

\begin{tabular}{llll}
\hline $\begin{array}{l}\text { Upregulation } \\
\text { miRNA }\end{array}$ & Fold change & $\begin{array}{l}\text { Downregulation } \\
\text { miRNA }\end{array}$ & Fold change \\
\hline hsa-miR-24-3p & 9.80 & hsa-miR-3613-3p & 0.14 \\
hsa-miR-93-5p & 9.73 & hsa-miR-1273 g-3p & 0.16 \\
hsa-miR-320a & 5.52 & hsa-miR-4668-5p & 0.38 \\
hsa-miR-320b & 4.62 & & \\
hsa-miR-191-5p & 3.73 & & \\
hsa-let-7b-5p & 3.43 & & \\
hsa-miR-342-3p & 2.62 & & \\
hsa-miR-92a-3p & 2.06 & & \\
\hline
\end{tabular}

The miRNAs with difference of fluorescence intensity higher than 1000 was selected

in miRNA sets between ex- and current smokers in the smokers and COPD groups.

\section{Verification of expression of dysregulated miRNAs in different cell types of PBMCs}

PBMCs consist mainly of T lymphocytes, B lymphocytes and monocytes. We therefore analyzed the expression of dysregulated miRNAs in the isolated different cell types including $\mathrm{CD}^{+}{ }^{+} \mathrm{T}$ cells, $\mathrm{CD} 8^{+} \mathrm{T}$ cells, $\mathrm{CD} 20^{+} \mathrm{B}$ cells and $\mathrm{CD}_{14}{ }^{+}$monocytes from PBMCs of smokers and COPD patients. MiR-24-3p was consistently expressed higher in monocytes. In smokers, miR-93-5p was mainly expressed

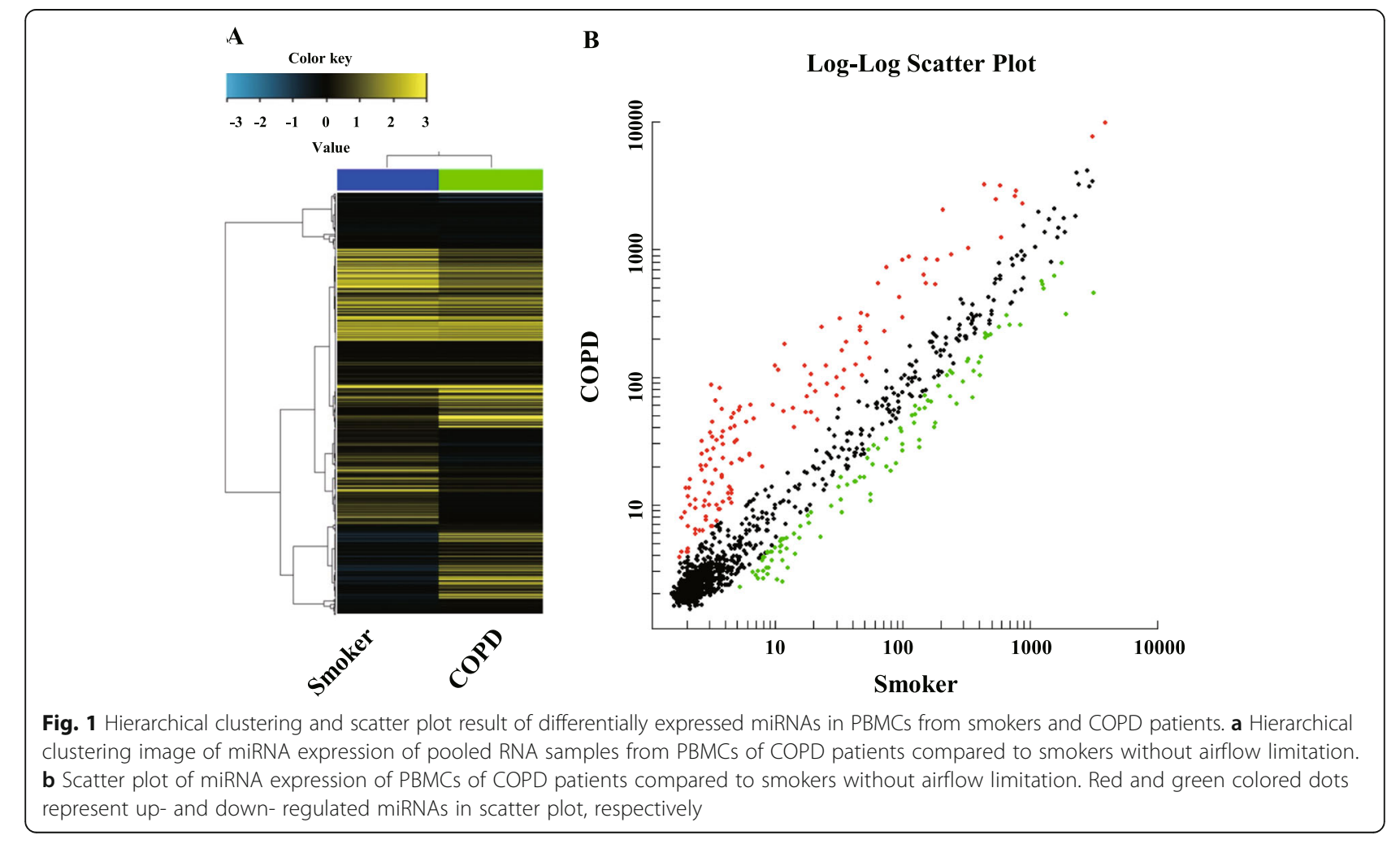


A
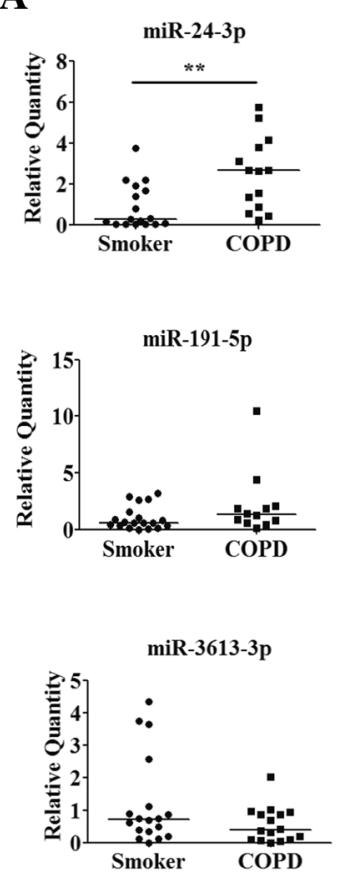

B

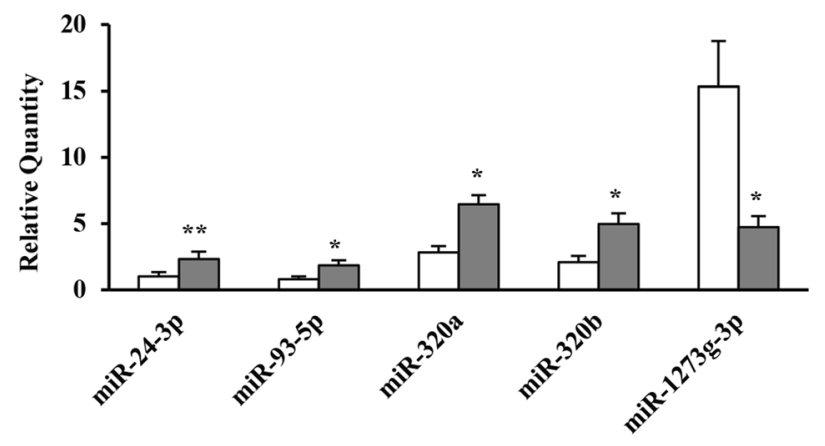

C

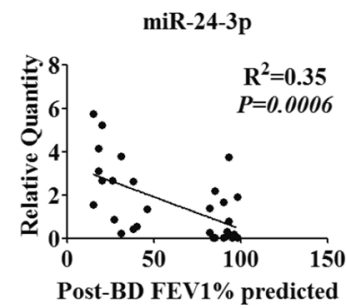

miR-320b

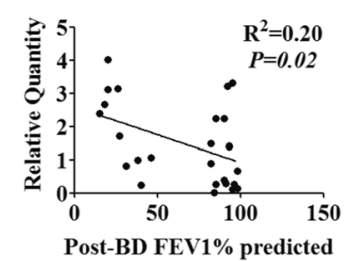

Fig. 2 (See legend on next page.)
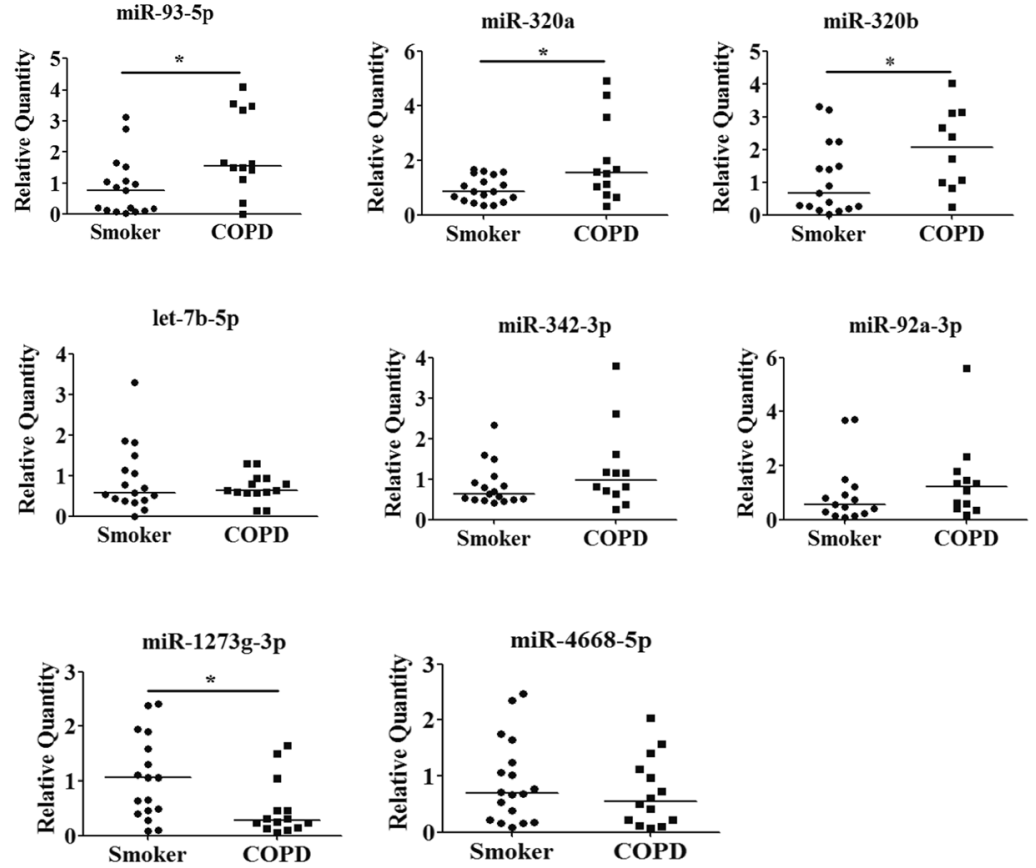

$\square$ Smoker

$\square$ COPD
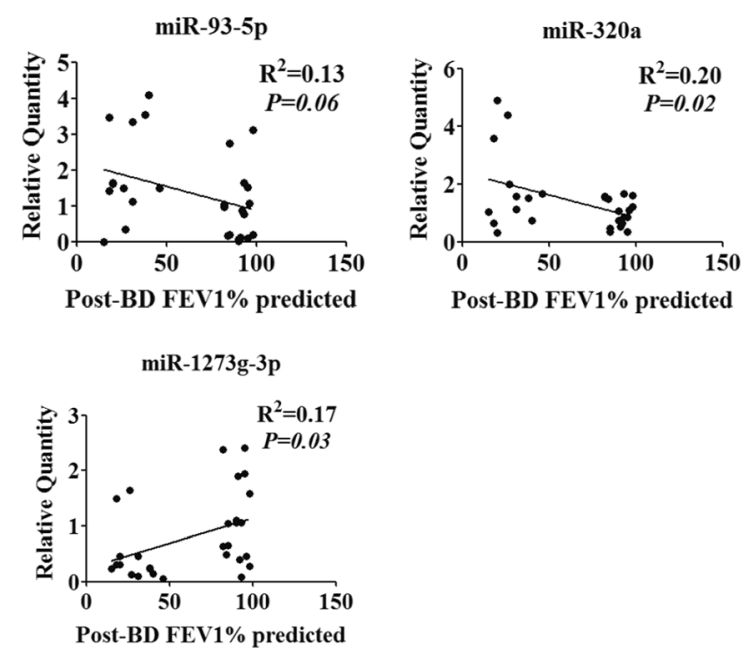
(See figure on previous page.)

Fig. 2 Validation of differentially expressed miRNAs. a Expression of selected miRNAs in PBMCs of smokers and COPD patients. GRT-PCR was performed on the same RNA samples (17smokers and 14 COPD patients) as microarray analysis. Data are presented as $2^{(-\sigma \sigma C t)}$ relative to U6. ${ }^{*} P<0.05,{ }^{* *} P<0.01$ compared with smokers by Mann Whitney $U$ test. b Relative abundance of differentially expressed miRNAs in PBMCs of smokers and COPD patients. ${ }^{*} P<0.05,{ }^{* *} P<0.01$ compared with smokers. c Correlation analysis between miRNA expression and FEV $1 \%$ predicted

in monocytes, while it was equally expressed in $\mathrm{CD} 4^{+}$ $\mathrm{T}$ cells, $\mathrm{CD}^{+} \mathrm{T}$ cells and monocytes in COPD patients. $\mathrm{CD} 8^{+} \mathrm{T}$ cells mainly contribute to the increased expression of miR-320a and miR-320b in COPD patients. In COPD patients, more miR-1273 g-3p was expressed in monocytes, suggesting that the expression was decreased in $\mathrm{CD}^{+} \mathrm{T}$ cells, $\mathrm{CD} 8^{+} \mathrm{T}$ cells and $\mathrm{CD} 20^{+} \mathrm{B}$ cells in COPD (Fig. 3).
mRNAs are dysregulated in PBMCs of COPD patients

We performed a parallel mRNA microarray study to compare the mRNA expression between the smokers and COPD patients. The results revealed a total of 1508 genes that differed in expression between the two groups (fold change $>2$; Fig. 4), where 164 mRNAs were upregulated and 137 were down-regulated when filtered by

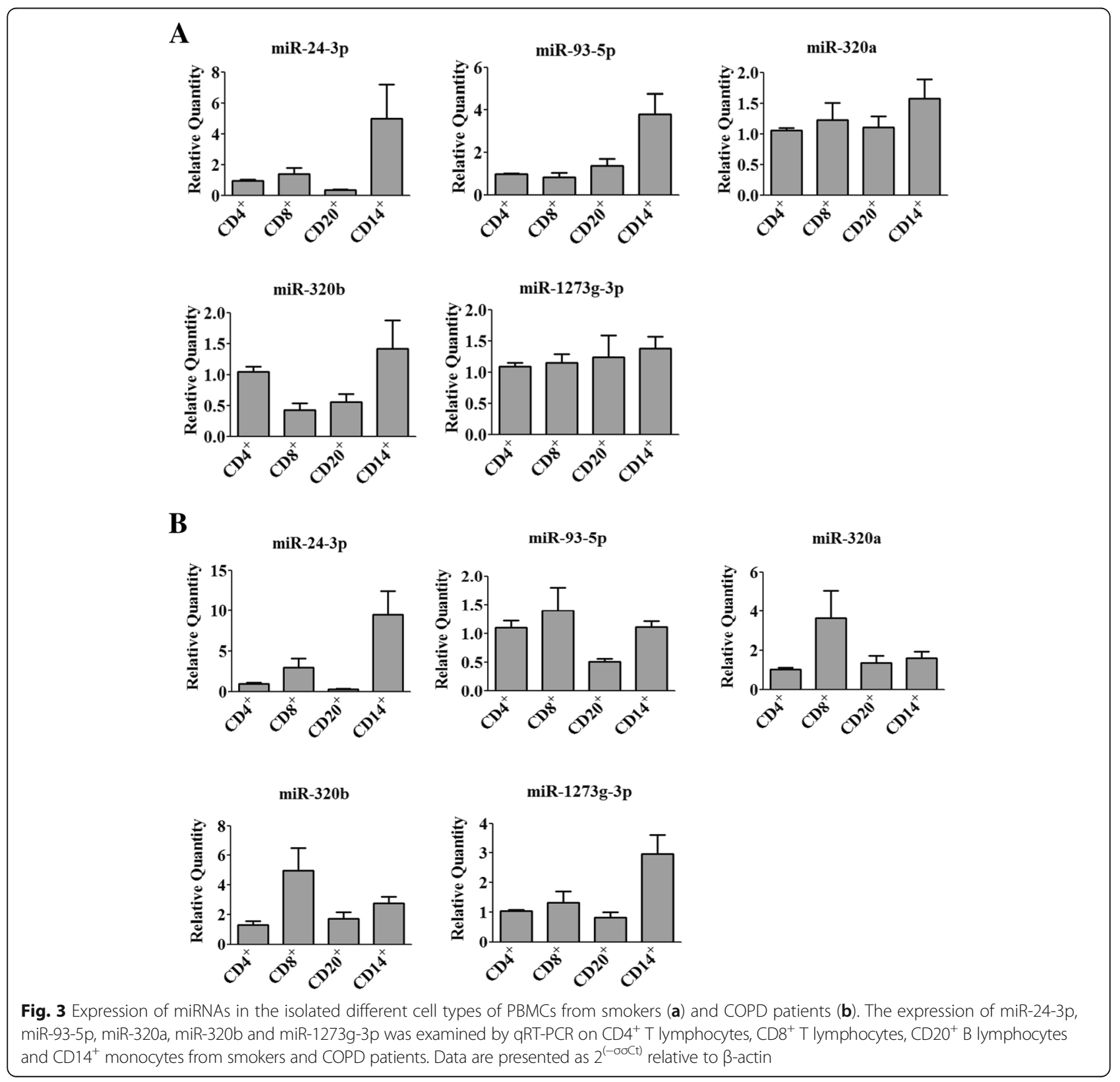


A

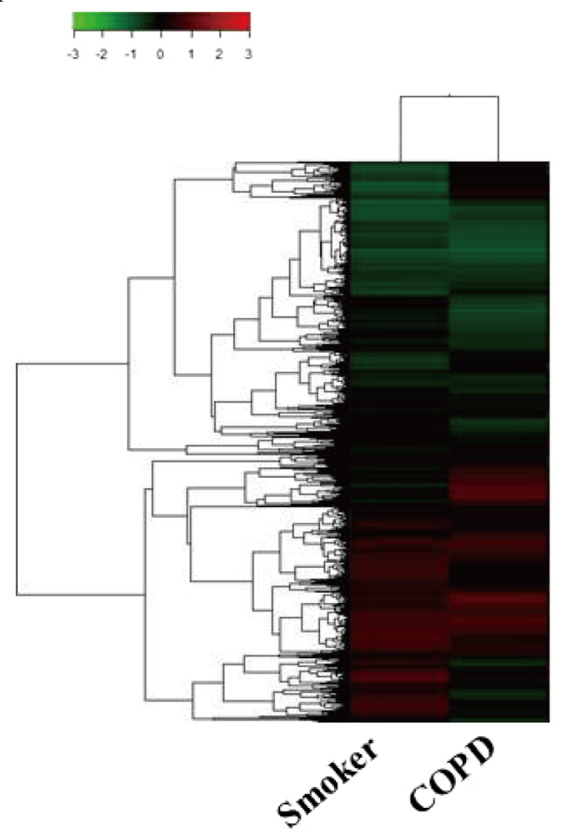

B

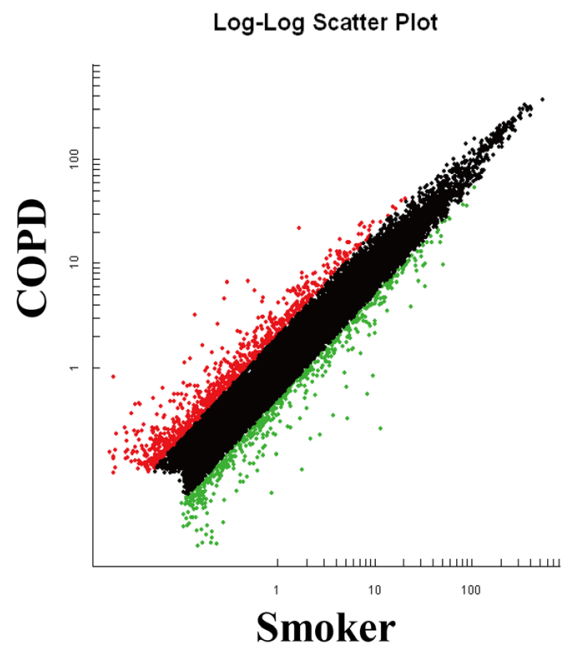

Fig. 4 Hierarchical clustering and scatter plot result of differentially expressed mRNAs in PBMCs from smokers and COPD patients. a Hierarchical clustering image of mRNA expression of pooled RNA samples from PBMCs of COPD patients compared to smokers without airflow limitation. b Scatter plot of mRNA expression of PBMCs of COPD patients compared to smokers without airflow limitation. Red and green colored dots represent up- and down- regulated mRNAs in scatter plot, respectively

fluorescence intensity difference higher than 1000 . Table 4 shows the 20 genes with the largest fold changes and their known biological functions.

\section{Regulation network of dysregulated miRNAs and mRNAs}

Based on the genes negatively correlated with miRNAs from microarray, the predicted regulatory network between the dysregulated miRNAs (that were validated) and mRNAs in COPD patients was then analyzed (Fig. 5, Table 5). The KEGG pathway enrichment analysis indicated that the NOD - like receptor (NLR) and Toll - like receptor (TLR) signaling pathway relevant to the pathogenesis of COPD were significantly enriched (Fig. 6). The involved genes include IL18/IL1B/TNF predicted to be regulated by miR-24-3p, and NLRP3/IL6/NFKBIA by miR-93-5p for NLR pathway. The genes CCL3/CCL4/ IL1B/TNF were predicted to be regulated by miR-24-3p, and IL6/CXCL10/NFKBIA by miR-93-5p for TLR pathway. The expression level of relevant predicted target genes of individual subjects were further validated by qRT-PCR. As shown in Fig. 7, the expression of IL18, IL1B, TNF, NFKBIA, CCL3 and CCL4 was validated, but not for NLRP3, IL6 and CXLC10.

\section{Discussion}

MicroRNAs play important regulatory roles in cell differentiation, cell cycle and apoptosis. Due to the role of multiple gene regulation, miRNAs have received much attention as biomarkers and target for novel therapeutics. In COPD therefore, the role of miRNAs in disease pathogenesis is an attractive area of research. For the first time, we conducted a comprehensive analysis of both miRNA and mRNA expression in PBMCs from subjects with COPD and compared their expression profiles to smokers without airflow limitation. We identified 137 differentially-expressed miRNAs in PBMCs from COPD subjects compared with smokers without COPD. Among the selected 11 miRNAs, the dysregulated expression of 5 miRNAs including miR-24-3p, miR-93-5p, miR-320a, miR-320b and miR-1273g-3p were validated by qPCR.

Of the miRNA investigated in this study, miR-24-3p is of considerable interest. It has been reported that miR24-3p was consistently upregulated during terminal differentiation of hematopoietic cells into a variety of lineages as well as during muscle and neuronal cell differentiation [20, 21]. MiR-24-3p might also function in cell proliferation $[22,23]$. Upregulation of miR-24 is associated with a decreased DNA damage response upon etoposide treatment in highly differentiated $\mathrm{CD}^{+} \mathrm{T}$ cells [24], and miR-24 is a negative regulator of classical macrophage activation by LPS [25]. In this study, we found increased expression of miR-24-3p mainly in the $\mathrm{T}$ lymphocytes and monocytes, which might in part 
Table 4 Top 10 dysregulated mRNAs in COPD patients compared with smokers without COPD

\begin{tabular}{|c|c|c|c|}
\hline Gene symbol & Gene name & $\begin{array}{l}\text { Fold change } \\
\text { COPD/Smokers }\end{array}$ & Function \\
\hline \multicolumn{4}{|l|}{ Upregulation } \\
\hline CD177 & CD177 molecule & 22.59 & Leukocyte migration \\
\hline MUC17 & Mucin 17 , cell surface associated & 21.54 & Extracellular matrix constituent \\
\hline IL1R2 & Interleukin 1 receptor, type II & 10.96 & Decoy receptor, inhibits the activity of IL-1 \\
\hline SARDH & Sarcosine dehydrogenase & 9.52 & Mitochondrial matrix \\
\hline EGR3 & Early growth response 3 & 6.75 & Positive regulation of endothelial cell proliferation \\
\hline AREG & Amphiregulin & 6.16 & EGF family, promote the growth of normal epithelial cells \\
\hline SLC6A2 & $\begin{array}{l}\text { Solute carrier family } 6 \\
\text { (neurotransmitter transporter, noradrenalin), member } 2\end{array}$ & 4.70 & Sodium symporter \\
\hline TMEM167A & Transmembrane protein 167A & 4.55 & Golgi apparatus \\
\hline KCNJ15 & $\begin{array}{l}\text { Potassium inwardly-rectifying channel, subfamily J, } \\
\text { member } 15\end{array}$ & 4.42 & Potassium channel activity \\
\hline $\mathrm{FCHO} 1$ & FCH domain only 1 & 4.39 & Clathrin-mediated endocytosis \\
\hline \multicolumn{4}{|c|}{ Downregulation } \\
\hline IL1A & Interleukin 1, alpha & -44.69 & Immune response \\
\hline IL6 & Interleukin 6 (interferon, beta 2) & -16.14 & Pro-inflammatory and anti-inflammatory role \\
\hline CXCL10 & Chemokine (C-X-C motif) ligand 10 & -15.08 & leukocyte chemotaxis \\
\hline TNF & Tumor necrosis factor & -11.79 & Inflammation, cause apoptosis \\
\hline CCL20 & Chemokine (C-C motif) ligand 20 & -7.94 & Lymphocytes chemotaxis \\
\hline CCL4 & Chemokine ( $\mathrm{C}-\mathrm{C}$ motif) ligand 4 & -6.19 & Leukocyte chemotaxis \\
\hline CCL3L3 & Chemokine (C-C motif) ligand 3-like 3 & -5.28 & Leukocyte chemotaxis \\
\hline C9orf7 & Chromosome 9 open reading frame 7 & -5.23 & Calcium channel activity \\
\hline IL1RN & Interleukin 1 receptor antagonist & -4.69 & Inhibition of the activities of IL-1 \\
\hline RNF19B & Ring finger protein $19 B$ & -4.17 & Cytotoxic effects of natural killer (NK) cells \\
\hline
\end{tabular}

contribute to the increased number of $\mathrm{CD}^{+} \mathrm{T}$ cells in the lung [26] and to the impairment of host defenses in the lower respiratory tract due to the smoke related changes in the phenotype of alveolar macrophages of COPD patients [27].

As for the rest of dysregulated miRNAs reported in this study, we did not find the relevant evidences involving the pathogenesis of COPD, which implies the discrepancy of the miRNA profile between PBMCs and lung tissues. These miRNAs were mainly found dysregulated in cancers and other disorders like autoimmune diseases. For example, miR-93-5p was identified as a potential biomarker of various types of cancer such as acute myeloid leukemia [28] and laryngeal squamous cell carcinoma [29]. This could be due to the link between miR-93 and promotion of tumor growth, angiogenesis and metastasis $[30,31]$. miR-93 is up-regulated in PBMCs from adult T-cell leukemia patients, and suppresses the expression of a tumor suppressor protein, tumor protein 53-induced nuclear protein 1 (TP53INP1) [32]. Four different transcripts have been reported in the database for miR-320 (miR-320a, b, c, and d) [33]. The seed region considered crucial for target binding remains the same for miR-320a, b, c, and d. The plasma level of miR-320a was found increased in patients with systemic lupus erythematosus (SLE) [34], and miR-320-3p is increased in the plasma of non-small cell lung cancer (NSCLC) patients [35]. The expression of miR-1273g-3p was found remarkably changed in Human Umbilical Vein Endothelial Cells (HUVECs) under acute glucose fluctuations, which was demonstrated to contribute to endothelial dysfunction and autophagy [36]. MiR-1273 expression is also increased in the pancreas of mouse model of pancreatic cancer [37].

By analyzing the regulation network between the dysregulated miRNA and mRNA, we predicted the negative regulatory role of miRNAs on a total of 36 over expressed and 61 under expressed mRNAs. The KEGG pathway enrichment analysis indicated that the NOD like receptor (NLR) signaling pathway and Toll - like receptor (TLR) signaling pathway are the top 2 pathways likely involved in the pathogenesis of COPD, with mir24-3p and miR-93-5p being predicted to regulate the relevant genes in both pathways. As down-stream factors of the NLR and TLR pathway, the mRNA levels of proinflammatory mediators including IL-18, IL-1 $\beta$, CCL3, 


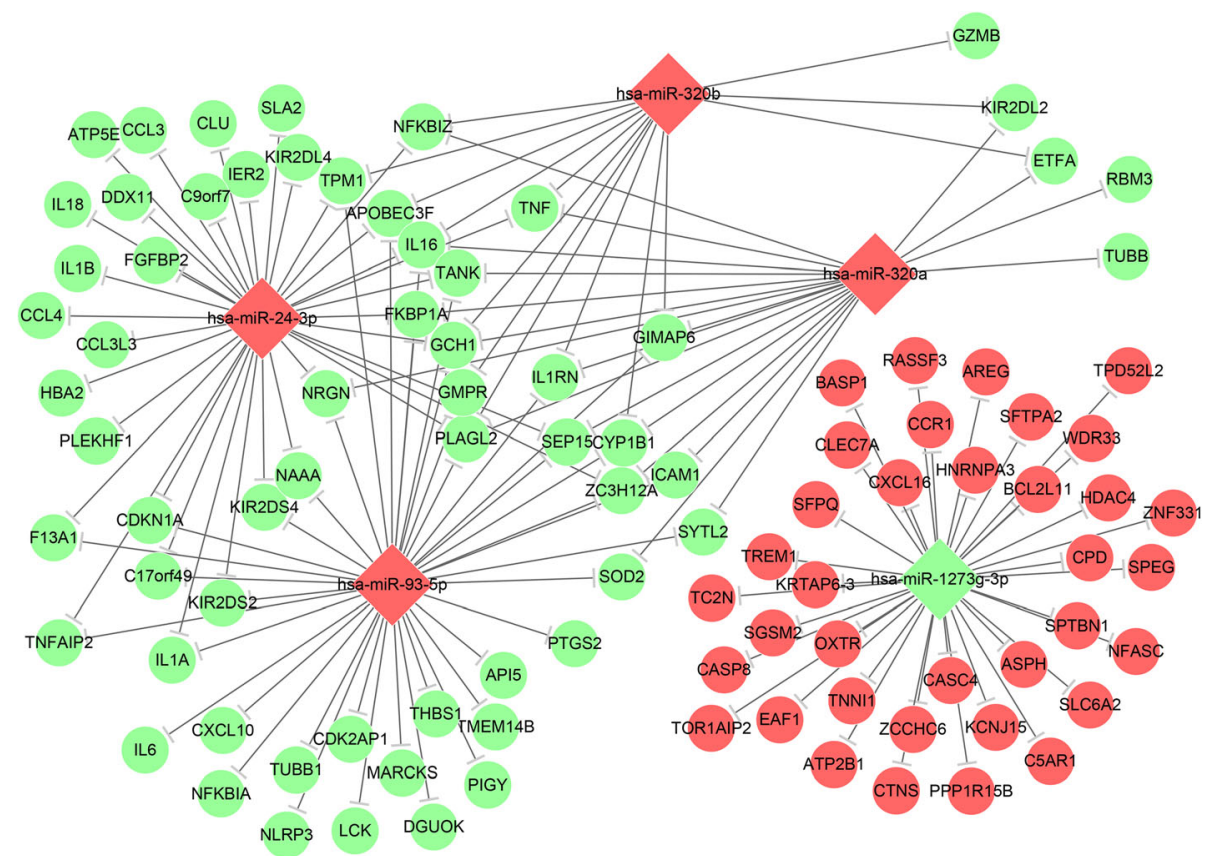

Fig. 5 Regulation network between miRNAs and mRNAs. The negatively regulation of miRNA on dysregulated mRNAs was predicted and the regulation network was drawn by using Cytoscape software. Red and green color represents up- and down-regulated genes, respectively

CCL4, and TNF were found down-regulated in PBMCs of COPD patients in this study. To the best of our knowledge, the down-regulation of these mRNAs in PBMCs of COPD patients has not been previously reported. In fact, a previous study performed on human peripheral lung tissue obtained from non-smokers, smokers and COPD patients revealed the similar trends of expression level of pro-inflammatory mediators, where the level of IL-8, IL-6, IL- $1 \beta$ and TNF- $\alpha$ showed the decreased trend in COPD patients compared with smokers [38]. Instead, most previous studies showed elevated expression levels of these mediators in serum or lung [39]. We suspect that certain changes in the cytokine expression profile may happen when the peripheral immune cells infiltrate the local inflammatory sites in the lung. The reduced expression of TLR2 has been found in the alveolar macrophages of smokers and COPD patients, which was associated with the impairment of host defenses in the lower respiratory tract [27]. Furthermore, decreased cytokine and chemokine mRNA expression in bronchoalveolar lavage cells from asymptomatic smokers has been reported [40].

In addition, NFKBIA predicted to be regulated by miR-93-5p- was the other down-regulated gene involved in enriched pathways. In unstimulated cells, NF- $k B$ is found in the cytoplasm in an inactive non-DNA binding form, associated with its inhibitory protein $\kappa B \alpha(\mathrm{I} \kappa \mathrm{B} \alpha$, coded by NFKBIA gene), IкB $\alpha$ degradation unmasks the nuclear localization signal present in NF- $\mathrm{kB}$, allowing it to enter the nucleus, bind DNA, and initiate gene transcription [41]. In the present study, the down-regulation of $\mathrm{I} \kappa \mathrm{B} \alpha$ supposedly trigger the activation of NF- $\kappa \mathrm{B}$, whose expression was also higher in PBMCs of COPD patients. The coexistence of decreased level of ІкB $\alpha$ and pro-inflammatory mediators in COPD patients was also reported on lung tissue [38]. Besides trigging the expression of pro-inflammatory mediators, NF- $\mathrm{kB}$ activation may also be related with the disordered apoptosis of $\mathrm{T}$ cell hybridoma cell line [42]. Thus, the decreased expression of ІкB $\alpha$ may contribute to the dysregulated apoptosis of $\mathrm{T}$ cells in COPD [43].

Overall, through miRNAs and mRNAs expression profiling in smokers and COPD patients, we identified the dysregulated miRNAs and mRNAs in PBMCs from COPD patients. We further analyzed the regulation network between miRNA and mRNA, where NLR and TLR was the most enriched pathways. Among them the regulation of IL-18, IL-1 $\beta$, TNF, CCL3 and CCL4 by miR-24-3p, and IkB $\alpha$ by miR-93-5p may provide the clue for potential investigations.

\section{Conclusions}

The expression of miRNA and mRNA were dysregulated in PBMCs of COPD patients compared with smokers without airflow limitation. The regulation network between the dysregulated miRNA and mRNA may provide potential therapeutic targets for COPD. 
Table 5 The pathway enrichment of dysregulated mRNAs regulated by miRNAs

\begin{tabular}{|c|c|c|c|c|}
\hline miRNA & Pathway & Genes & Gene Ratio & $P$ Value \\
\hline \multirow[t]{10}{*}{ miR-24-3p } & Rheumatoid arthritis & IL1A/CCL3/CCL3L3/IL18/IL1B/TNF & $6 / 16$ & $2.37 E-06$ \\
\hline & African trypanosomiasis & HBA2/IL18/IL1B/TNF & $4 / 16$ & $2.47 \mathrm{E}-05$ \\
\hline & Cytokine-cytokine receptor interaction & IL1A/CCL3/CCL4/CCL3L3/IL18/IL1B/TNF & $7 / 16$ & 2.53E-05 \\
\hline & Malaria & HBA2/IL18/IL1B/TNF & $4 / 16$ & $5.74 \mathrm{E}-05$ \\
\hline & Toll-like receptor signaling pathway & CCL3/CCL4/IL1B/TNF & $4 / 16$ & 0.000645 \\
\hline & Chagas disease (American trypanosomiasis) & CCL3/CCL3L3/IL1B/TNF & $4 / 16$ & 0.000645 \\
\hline & Graft-versus-host disease & IL1A/IL1B/TNF & $3 / 16$ & 0.000741 \\
\hline & Type I diabetes mellitus & IL1A/IL1B/TNF & $3 / 16$ & 0.000743 \\
\hline & Cytosolic DNA-sensing pathway & CCL4/IL18/IL1B & $3 / 16$ & 0.001268 \\
\hline & NOD-like receptor signaling pathway & IL18/IL1B/TNF & $3 / 16$ & 0.001331 \\
\hline \multirow[t]{10}{*}{ miR-320a } & Natural killer cell mediated cytotoxicity & ICAM1/KIR2DL2/TNF & $3 / 8$ & 0.018267 \\
\hline & African trypanosomiasis & ICAM1/TNF & $2 / 8$ & 0.018267 \\
\hline & Graft-versus-host disease & KIR2DL2/TNF & $2 / 8$ & 0.018382 \\
\hline & Malaria & ICAM1/TNF & $2 / 8$ & 0.01936 \\
\hline & RIG-I-like receptor signaling pathway & TANKTNF & $2 / 8$ & 0.029778 \\
\hline & Antigen processing and presentation & KIR2DL2/TNF & $2 / 8$ & 0.029845 \\
\hline & Rheumatoid arthritis & ICAM1/TNF & $2 / 8$ & 0.035321 \\
\hline & Folate biosynthesis & $\mathrm{GCH} 1$ & $1 / 8$ & 0.072257 \\
\hline & Asthma & TNF & $1 / 8$ & 0.150454 \\
\hline & Graft-versus-host disease & GZMB/KIR2DL2/TNF & $3 / 7$ & 0.000379 \\
\hline \multirow[t]{10}{*}{ miR-320b } & Graft-versus-host disease & GZMB/KIR2DL2/TNF & $3 / 7$ & 0.000379 \\
\hline & Natural killer cell mediated cytotoxicity & GZMB/KIR2DL2/TNF & $3 / 7$ & 0.005999 \\
\hline & Allograft rejection & GZMB/TNF & $2 / 7$ & 0.008916 \\
\hline & Type I diabetes mellitus & GZMB/TNF & $2 / 7$ & 0.008916 \\
\hline & Antigen processing and presentation & KIR2DL2/TNF & $2 / 7$ & 0.019996 \\
\hline & Hypertrophic cardiomyopathy (HCM) & TNF/TPM1 & $2 / 7$ & 0.019996 \\
\hline & Dilated cardiomyopathy & TNF/TPM1 & $2 / 7$ & 0.020091 \\
\hline & Folate biosynthesis & GCH1 & $1 / 7$ & 0.049597 \\
\hline & Asthma & TNF & $1 / 7$ & 0.117955 \\
\hline & African trypanosomiasis & TNF & $1 / 7$ & 0.117955 \\
\hline \multirow[t]{11}{*}{ miR-93-5p } & Malaria & IL6/ICAM1/THBS1 & $3 / 22$ & 0.020688 \\
\hline & Cytosolic DNA-sensing pathway & IL6/CXCL10/NFKBIA & $3 / 22$ & 0.020688 \\
\hline & NOD-like receptor signaling pathway & NLRP3/IL6/NFKBIA & $3 / 22$ & 0.020688 \\
\hline & RIG-I-like receptor signaling pathway & CXCL10/NFKBIA/TANK & $3 / 22$ & 0.022958 \\
\hline & Leishmaniasis & IL1A/NFKBIA/PTGS2 & $3 / 22$ & 0.022958 \\
\hline & Rheumatoid arthritis & IL6/ICAM1/IL1A & $3 / 22$ & 0.036896 \\
\hline & Toll-like receptor signaling pathway & IL6/CXCL10/NFKBIA & $3 / 22$ & 0.041751 \\
\hline & African trypanosomiasis & IL6/ICAM1 & $2 / 22$ & 0.041751 \\
\hline & Prion diseases & IL6/IL1A & $2 / 22$ & 0.041751 \\
\hline & Bladder cancer & CDKN1A/THBS1 & $2 / 22$ & 0.045977 \\
\hline & Malaria & IL6/ICAM1/THBS1 & $3 / 22$ & 0.020688 \\
\hline
\end{tabular}



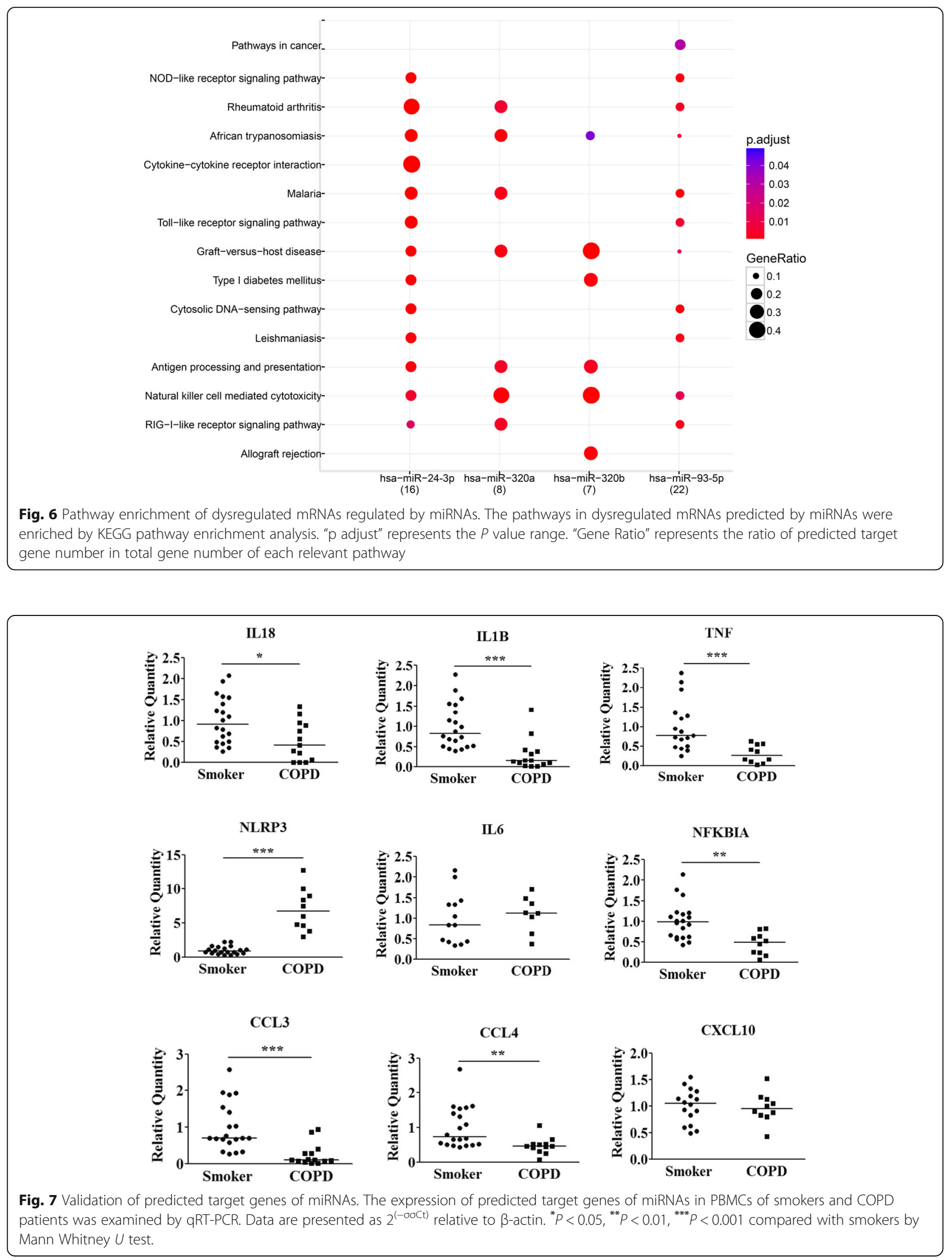


\section{Abbreviations}

COPD: Chronic obstructive pulmonary disease; NLR: NOD - like receptor; PBMC: Peripheral blood mononuclear cell; TLR: Toll - like receptor

\section{Acknowledgements}

The authors acknowledge Dr. Yong-Ping Shao and Dr. Wuyuan Lu for their critical revise on manuscript and valuable suggestions on study.

\section{Funding}

This study was supported by National Natural Science Foundation of China (31501044), Natural Science Basic Research Plan in Shaanxi Province of China (2015JQ3066), Sci-tech Research and Development Project of Shaanxi Province of China (2016KW-026), Fundamental Research Funds for the Central Universities (xjj2014088), the Scientific Research Foundation for the Returned Overseas Chinese Scholars, State Education Ministry and the Richard and Edith Strauss Canada Foundation. This work was supported in part by Project 985 of Xi'an Jiaotong University.

\section{Availability of data and materials}

Not applicable.

\section{Authors' contributions}

XD collected blood samples. XQ isolated PBMCs, performed qRT-PCR and cell culture and analyzed the data. WW isolated part of PBMCs. CL helped collecting blood samples. YL performed part of cell culture. DX coordinated the collection of blood samples. CJB revised the manuscript. DS collected blood samples. YC designed the study and drafted the manuscript. All authors read and approved the final manuscript.

\section{Competing interests}

The authors declare that there have no competing interests.

\section{Consent for publication}

Not applicable.

\section{Ethics approval and consent to participate}

The experimental procedures were performed with ethical approval from the Research Ethics Boards of The First Affiliated Hospital, Xi'an Jiaotong University (2015-015)

\section{Author details}

${ }^{1}$ Center for Translational Medicine, The Key Laboratory of Biomedical Information Engineering of Ministry of Education, School of Life Science and Technology and Frontier Institute of Science and Technology, Xi'an Jiaotong University, Xi'an 710049, Shaanxi province, China. ${ }^{2}$ Department of Respiration, The First Affiliated Hospital, Xi'an Jiaotong University, Xi'an 710061, Shaanxi province, China. ${ }^{3}$ Meakins-Christie Laboratories and Respiratory Division, Health Centre and Department of Medicine, McGill University, Montreal, QC, Canada.

Received: 31 May 2016 Accepted: 9 December 2016

Published online: 05 January 2017

\section{References}

1. Pauwels RA, Rabe KF. Burden and clinical features of chronic obstructive pulmonary disease (COPD). Lancet. 2004;364:613-20.

2. Cosio MG, Majo J, Cosio MG. Inflammation of the airways and lung parenchyma in COPD: role of T cells. Chest. 2002;121:160S-5S.

3. Stankiewicz W, Dabrowski MP, Chcialowski A, Plusa T. Cellular and cytokine immunoregulation in patients with chronic obstructive pulmonary disease and bronchial asthma. Mediators Inflamm. 2002;11:307-12.

4. Cosio MG, Saetta M, Agusti A. Immunologic aspects of chronic obstructive pulmonary disease. N Engl J Med. 2009;360:2445-54.

5. Bartel DP. MicroRNAs: target recognition and regulatory functions. Cell. 2009;136:215-33

6. Faruq O, Vecchione A. microRNA: Diagnostic Perspective. Front Med (Lausanne). 2015;2:51.

7. Booton R, Lindsay MA. Emerging role of MicroRNAs and long noncoding RNAs in respiratory disease. Chest. 2014;146:193-204.
8. Mansoori B, Mohammadi A, Shirjang S, Baradaran B. Micro-RNAs: The new potential biomarkers in cancer diagnosis, prognosis and cancer therapy. Cell Mol Biol (Noisy-le-Grand). 2015;61:1-10.

9. Ezzie ME, Crawford M, Cho JH, Orellana R, Zhang S, Gelinas R, et al. Gene expression networks in COPD: microRNA and mRNA regulation. Thorax. 2012;67:122-31.

10. Van Pottelberge GR, Mestdagh $P$, Bracke KR, Thas O, van Durme YM, Joos GF, et al. MicroRNA expression in induced sputum of smokers and patients with chronic obstructive pulmonary disease. Am J Respir Crit Care Med. 2011;183:898-906

11. Savarimuthu Francis SM, Davidson MR, Tan ME, Wright CM, Clarke BE, Duhig EE, et al. MicroRNA-34C is associated with emphysema severity and modulates SERPINE1 expression. BMC Genomics. 2014;15:88.

12. Sato T, Liu X, Nelson A, Nakanishi M, Kanaji N, Wang X, et al. Reduced miR146a increases prostaglandin $\mathrm{E}(2)$ in chronic obstructive pulmonary disease fibroblasts. Am J Respir Crit Care Med. 2010;182:1020-9.

13. Lewis A, Riddoch-Contreras J, Natanek SA, Donaldson A, Man WD, Moxham J, et al. Downregulation of the serum response factor/miR-1 axis in the quadriceps of patients with COPD. Thorax. 2012;67:26-34.

14. Ma J, Lin Y, Zhan M, Mann DL, Stass SA, Jiang F. Differential miRNA expressions in peripheral blood mononuclear cells for diagnosis of lung cancer. Lab Invest. 2015;95:1197-206.

15. Leidinger $P$, Keller $A$, Borries $A$, Huwer $H$, Rohling $M$, Huebers J, et al. Specific peripheral miRNA profiles for distinguishing lung cancer from COPD. Lung Cancer. 2011;74:41-7.

16. Ren RJ, Zhang YF, Dammer EB, Zhou Y, Wang LL, Liu XH, et al. Peripheral Blood MicroRNA Expression Profiles in Alzheimer's Disease: Screening, Validation, Association with Clinical Phenotype and Implications for Molecular Mechanism. Mol Neurobiol. 2016;53:5772-81.

17. Yang M, Ye L, Wang B, Gao J, Liu R, Hong J, et al. Decreased miR-146 expression in peripheral blood mononuclear cells is correlated with ongoing islet autoimmunity in type 1 diabetes patients 1miR-146. J Diabetes. 2015;7:158-65.

18. Liu D, Zhao H, Zhao S, Wang X. MicroRNA expression profiles of peripheral blood mononuclear cells in patients with systemic lupus erythematosus. Acta Histochem. 2014;116:891-7.

19. Huang DW, Sherman BT, Tan Q, Collins JR, Alvord WG, Roayaei J, et al. The DAVID Gene Functional Classification Tool: a novel biological modulecentric algorithm to functionally analyze large gene lists. Genome Biol. 2007;8:R183.

20. Xu W, Liu M, Peng X, Zhou P, Zhou J, Xu K, et al. miR-24-3p and miR-27a-3p promote cell proliferation in glioma cells via cooperative regulation of MXI1. Int J Oncol. 2013;42:757-66.

21. Fukuda $Y$, Kawasaki $H$, Taira K. Exploration of human miRNA target genes in neuronal differentiation. Nucleic Acids Symp Ser (Oxf). 2005;49:341-2.

22. Qin W, Shi Y, Zhao B, Yao C, Jin L, Ma J, et al. miR-24 regulates apoptosis by targeting the open reading frame (ORF) region of FAF1 in cancer cells. PLoS One. 2010;5:e9429.

23. Cheng AM, Byrom MW, Shelton J, Ford LP. Antisense inhibition of human miRNAs and indications for an involvement of miRNA in cell growth and apoptosis. Nucleic Acids Res. 2005;33:1290-7.

24. Brunner S, Herndler-Brandstetter D, Arnold CR, Wiegers GJ, Villunger A, Hackl M, et al. Upregulation of miR-24 is associated with a decreased DNA damage response upon etoposide treatment in highly differentiated CD8(+) T cells sensitizing them to apoptotic cell death. Aging Cell. 2012;11:579-87.

25. Fordham JB, Naqvi AR, Nares S. miR-24 Regulates Macrophage Polarization and Plasticity. J Clin Cell Immunol. 2015;6:362.

26. O'Donnell R, Breen D, Wilson S, Djukanovic R. Inflammatory cells in the airways in COPD. Thorax. 2006;61:448-54.

27. Droemann D, Goldmann T, Tiedje T, Zabel P, Dalhoff K, Schaaf B. Toll-like receptor 2 expression is decreased on alveolar macrophages in cigarette smokers and COPD patients. Respir Res. 2005;6:68.

28. Zhi F, Cao X, Xie X, Wang B, Dong W, Gu W, et al. Identification of circulating microRNAs as potential biomarkers for detecting acute myeloid leukemia. PLoS One. 2013;8:e56718.

29. Ayaz L, Gorur A, Yaroglu HY, Ozcan C, Tamer L. Differential expression of microRNAs in plasma of patients with laryngeal squamous cell carcinoma: potential early-detection markers for laryngeal squamous cell carcinoma. J Cancer Res Clin Oncol. 2013;139:1499-506.

30. Fang L, Deng Z, Shatseva T, Yang J, Peng C, Du WW, et al. MicroRNA miR-93 promotes tumor growth and angiogenesis by targeting integrin-beta8. Oncogene. 2011;30:806-21. 
31. Fang L, Du WW, Yang W, Rutnam ZJ, Peng C, Li H, et al. MiR-93 enhances angiogenesis and metastasis by targeting LATS2. Cell Cycle. 2012;11:4352-65.

32. Yeung ML, Yasunaga J, Bennasser Y, Dusetti N, Harris D, Ahmad N, et al. Roles for microRNAs, miR-93 and miR-130b, and tumor protein 53-induced nuclear protein 1 tumor suppressor in cell growth dysregulation by human T-cell lymphotrophic virus 1. Cancer Res. 2008;68:8976-85.

33. Sepramaniam S, Armugam A, Lim KY, Karolina DS, Swaminathan P, Tan JR, et al. MicroRNA 320a functions as a novel endogenous modulator of aquaporins 1 and 4 as well as a potential therapeutic target in cerebral ischemia. J Biol Chem. 2010;285:29223-30.

34. Wang $H$, Peng W, Ouyang $X, L i W$, Dai Y. Circulating microRNAs as candidate biomarkers in patients with systemic lupus erythematosus. Transl Res. 2012;160:198-206.

35. Sanfiorenzo C, llie MI, Belaid A, Barlesi F, Mouroux J, Marquette CH, et al. Two panels of plasma microRNAs as non-invasive biomarkers for prediction of recurrence in resectable NSCLC. PLoS One. 2013;8:e54596.

36. Guo J, Sang Y, Yin T, Wang B, Yang W, Li X, et al. miR-1273g-3p Participates in Acute Glucose Fluctuations Induced Autophagy, Dysfunction And Proliferation Attenuation in Human Umbilical Vein Endothelial Cells. Am J Physiol Endocrinol Metab. 2016;10:E734-43. doi:10.1152/ajpendo.00444.2015.

37. Rachagani S, Macha MA, Menning MS, Dey P, Pai P, Smith LM, et al. Changes in microRNA (miRNA) expression during pancreatic cancer development and progression in a genetically engineered KrasG12D;Pdx1Cre mouse (KC) model. Oncotarget. 2015;6:40295-309.

38. Szulakowski P, Crowther AJ, Jimenez LA, Donaldson K, Mayer R, Leonard TB, et al. The effect of smoking on the transcriptional regulation of lung inflammation in patients with chronic obstructive pulmonary disease. Am J Respir Crit Care Med. 2006;174:41-50.

39. de Boer WI. Perspectives for cytokine antagonist therapy in COPD. Drug Discov Today. 2005;10:93-106.

40. Meuronen A, Majuri ML, Alenius $H$, Mantyla T, Wolff H, Piirila P, et al. Decreased cytokine and chemokine mRNA expression in bronchoalveolar lavage in asymptomatic smoking subjects. Respiration. 2008;75:450-8.

41. Baldwin Jr AS. The NF-kappa B and I kappa B proteins: new discoveries and insights. Annu Rev Immunol. 1996;14:649-83.

42. Barkett M, Gilmore TD. Control of apoptosis by Rel/NF-kappaB transcription factors. Oncogene. 1999;18:6910-24.

43. Hodge S, Hodge G, Holmes M, Reynolds PN. Increased airway epithelial and T-cell apoptosis in COPD remains despite smoking cessation. Eur Respir J. 2005;25:447-54

\section{Submit your next manuscript to BioMed Central and we will help you at every step:}

- We accept pre-submission inquiries

- Our selector tool helps you to find the most relevant journal

- We provide round the clock customer support

- Convenient online submission

- Thorough peer review

- Inclusion in PubMed and all major indexing services

- Maximum visibility for your research

Submit your manuscript at www.biomedcentral.com/submit

) Biomed Central 